\section{Simple sterile packaging}

Hygopac View, from Dürr Dental, is an intelligent rotary sealing device which ensures instruments can be reliably packaged, stored and validated in sterile conditions. Obviously, there is no room for error when it comes to reprocessing instruments that have come into contact with patients' skin, mucous membranes or blood. Every step in the packaging process must be tested, but also simple to operate, bearing in mind the repetitive nature of such a task. Hygopac View has an intuitive touch display, which takes the operator through the step-by-step process,

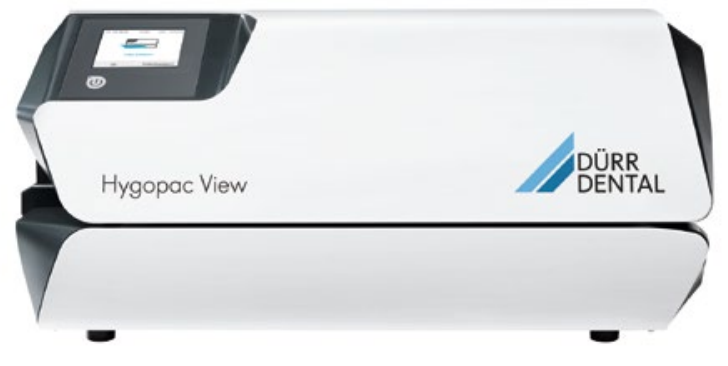

providing the necessary validation once completed. The sealing temperature, contact pressure and sealing speed are continuously recorded, checked and saved to an SD card or to the network via the VistaSoft Monitor software. This allows effortless processing of the data for documentation within a quality management system. In the event of a practice inspection, the information is available immediately.

As well as offering great flexibility and reliability in terms of process documentation, HygoPac View also boasts an exceptionally efficient design, which is a bonus as it's often used in areas that have limited space.

\section{Adaption and evolution}

Modern dentistry is all about adapting to patient demands and evolving with the times. Carestream Dental has remained at the cutting-edge of the profession for several decades now and it is committed to delivering technologies that simplify every day workflows.

The CS Adapt module is one such solution. It enables dentists to define how extraoral images look with several filters available to suit their individual preferences. The easy-to-use filter library offers six options for panoramic images and four options for cephalometric images, with the capacity to create customised settings and save any favourites.

All this serves to reduce the risk of misdiagnosis and make every day routines simpler for the professional.

Compatible with the CS 8100 family of imaging units, as well as the CS 9000/CS 9300 CBCT machine, CS Adapt allows every practitioner to work in the way they want to.

Contact the friendly team at Carestream Dental and start adapting your images to you today. For more information contact Carestream Dental on 08001699692 or visit www.carestreamdental.co.uk.

\section{Keep your patients smiling with confidence}

Solvay Dental 360 has custom-developed Ultaire AKP as a modern, metal-free alternative to traditional removable partial dentures (RPDs).

Specifically designed to fit the digital workflow, this new generation material offers an accurate fit with superior retention and aesthetics. Ultaire AKP is lightweight yet it does not distort and is remarkably strong and resilient. It also has a bone-like feel and is highly compliant, which makes it pleasant and comfortable to wear.

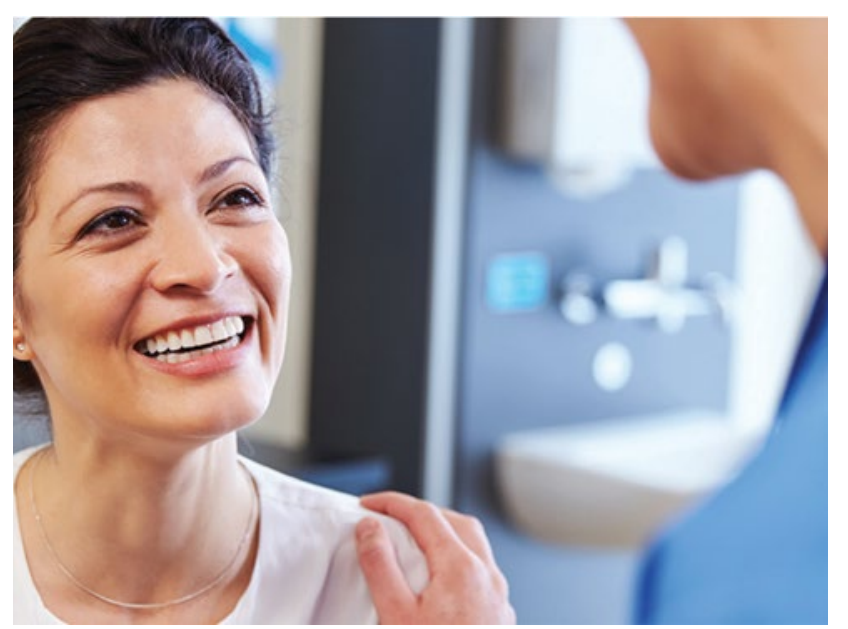

Biocompatible with absolutely no metallic taste, partial dentures made from this long-life polymer will keep your patients smiling with confidence for years to come.

To book a Solvay Dental 360 Professional Lunch and Learn or to find more information Ultaire AKP and Dentivera milling discs, visit www.solvaydental360.com.

\section{Top quality dental implant outcomes}

The Implantmed unit from W\&H provides you with the tools you need to ensure top quality dental implant outcomes. From its intuitive touch screen to automatic thread cutter and torque control functions, this unit has been designed to give surgeons exactly what they need. Intelligently designed and easy to use, the Implantmed can be fitted with the Osstell ISQ module, giving you extremely precise and reliable stability measurements.

Stability is critical to implant success. With the easy-to-read measurement provided by the Osstell ISQ module, you can monitor the osseointegration process through secondary stability readings.

Find out more about the award-winning Implantmed by contacting W\&H today. Visit www. wh.com/en_uk, call 01727874990 or email office.uk@wh.com.

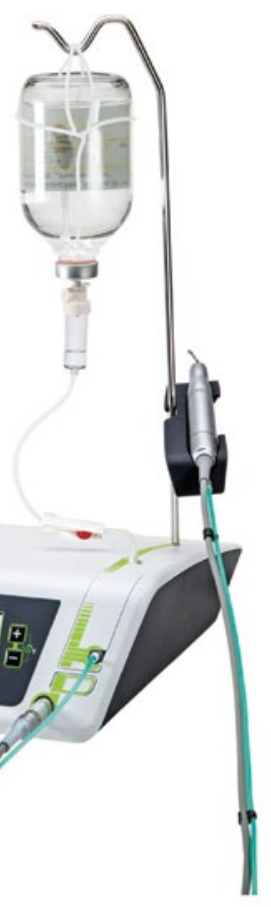

